\title{
Hall impact on the MHD fluid flow and heat transfer with uniform radial electric field due to a stretching rotating disk
}

\author{
Nihan Uygun (1) \\ Mathematics Department, University of Bolu Abant Izzet Baysal, Bolu, Turkey
}

\begin{abstract}
In the current paper, the steady flow of an incompressible electrically conducting fluid and heat transfer are studied. In these, we consider the Hall effect over an infinite stretching rotating disk in presence of a magnetic field. Navier-Stokes equations, Maxwell equation and energy equation have been modified in the presence of the Hall impact. Moreover, the uniform magnetic field, and the radial electric field are applied. With the help of the usual similarity transformations, these modified equations are simplified to a set of nonlinear ordinary differantial equations. Numerical solutions of the equations are obtained by using the Chebyshev collocation technique for different values of the entire of the physical parameters. The accuracy of the method is verified by comparing with the results in the literature. The influences of Hall parameter in these equations system are depicted graphically and analyzed.
\end{abstract}

Mathematics Subject Classification (2020). 76W05, 76E07

Keywords. Hall impact, incompressible flow, magnetic field, electric field, rotating-disk flow

\section{Introduction}

In the literature, the investigation of MHD fluid flow and heat transfer over a rotating disk flow has been placed great importance, thus having been investigated experimentally, numerically as well as theoretically. The flow is the rotating disk flow and also, using apparatus in machine similar to disk shape, because of these the hydrodynamic flow depends on a stretchable disk directly or indirectly. In these respects, stretchable disks are significant in many applications such as crystal growth process [1], extrusion process in plastic and metal industries [2], hot rolling [11], aerodynamical applications [13], turbomachinery [29], rotating machinery [29], oceanography [29], gas turbine rotors [29], glass fiber and paper production [17], cooling of metallic sheets or electronic chips [17], computer storage devices [22], lubrication [25], thermal-power generating systems [25], medical equipment [25], air cleaning machines [25], nuclear reactors [24]. In this paper, Hall impact is explored for the MHD fluid flow and heat transfer over stretching rotating disk with uniform radial electric field.

Email address: nuygun@ibu.edu.tr

Received: 12.05.2020; Accepted: 29.11.2021 
Pioneering work of fluid flow was carried out by Von Kármán [20], triggered further studies on an infinite rotating disk. The steady motion of an incompressible viscous fluid was investigated by numerical Cochran [8] and asymptotical Benton [6] means. Crane [10] obtained a similarity solution in closed analytical form for steady two-dimensional incompressible boundary layer flow caused by stretching of a sheet. Studies of Altan, Oh \& Gegel [2], Fisher [15] and Tadmor [32], the flow induced by a moving boundary, are important in the extrusion processes in plastic and metal industries. Wang [40] extented stretching boundary problem to a three-dimensional case. An exact solution for the steadystate Navier-Stokes equation in cylindrical coordinates was obtained by Fang [13]. Fang and Zhang studied the flow between two stretching disks in [14]. Watson and Wang [41] studied the unsteady flow over a rotating disk.

Millsaps \& Pohlhausen [23] considered the heat transfer problem on the rotating infinite disk. Following this study, the heat transfer on a flat plate was analyzed by Sparrow \& Gregg [31] for Prandtl numbers. Sparrow \& Cess [30], Riley [26], Kumar, Thacker \& Watson [21] studied the effects of magnetic field to the heat transfer over a infinite rotating disk. Many researchers followed them in [9, 19, 28,30,33-39]. Ultimately, effects of the uniform radial electric field have been considered in some works in the literature $[33,38,39]$. In these papers, Turkyilmazoglu [33] has triggered in his works examining effects of radial electric field on the flow over a infinite rotating disk. After this work, Uygun [38-39] studied analysis of Hall current and effects of uniform electric field on the MHD and heat transfer due to a rotating disk.

The electromagnetic force, taken upright to both the electric and magnetic fields, is generated in the flow, because of the motion of an electrically conducting fluid in a magnetic field. Due to the presence of this force the charged molecules to transpose in its own direction and generate an electrical current density termed as the Hall current. Normally, due to little and moderate values of the magnetic field, the impact of Hall current is disregarded in enforcing the Ohm's law whereas the influence of Hall current cannot be ignored for a strong magnetic field.

The influence of the electromagnetic force is noticeable when a strong magnetic field is applied as it was stated by Cramer and Pai [9]. Therefore, when the strength of the magnetic field is very strong then hall current cannot be neglected and Ohm's law also needs modification to include hall current effects. The Hall parameter is important and it has a significant effect on the magnitude and the direction of a current density, and consequently, on the magnetic force term.

In the literature, applying of Ohm's law in the analysis, most of the studies the Hall term is neglected for small or moderate values of the magnetic field, however in some works the Hall effect has been taken into consideration. To the best of our knowledge, Attia has initiated in [4] his study examining the Hall effect on the flow over an infinite rotating disk. The study has been continued by Attia \& Aboul-Hassan in [5], by Siddiqui, Rana \& Naseer in [28] and by Uygun in $[38,39]$. The case without the Hall effect on a rotating infinite disk has been investigated in $[3,7,9,12,19,21,23,26,28,30,31,33-36]$.

In the current paper, Hall current on the steady hydromagnetic flow of viscous, incompressible fluids over stretching disk is examined with induced uniform electric field in the radial direction. This problem is an extension of well-known von Kármán viscous pump problem to the configuration with a stretchable disk with or without rotation. In this work, an external uniform magnetic field is imposed for the normal direction, and a radial electric field is produced by an electric potential. Because of the existence of a uniform radial electric field at the infinity, a radial pressure gradient is generated. In the disk flow, Magnetic Reynolds number is assumed to be negligible. The Navier-Stokes equation, the Maxwell equation and the energy equation have been modified for considered the presence of uniform radial electric field, magnetic field, and also Hall effect. The governing partial 
differential equations have been transformed into the form of non-linear ordinary differential equations by using Kármán's similarity transformations. The system of equations generated by the Navier-Stokes, Maxwell and energy equations has been solved using spectral Chebyshev collocation technique for varying values of Hall parameters, radial electric parameters, rotation parameters, Eckert numbers, and for a fixed magnetic interaction number. Impacts of the Hall parameters in these equations are analyzed.

The rest of the paper relies on the following strategy. In Section 2 equations governing the motion are derived. Section 3 presents the results and discussion. Finally, Section 4 contains the conclusions drawn from the present results.

\section{Formulation of the problem}

We consider three-dimensional, MHD laminar flow of a viscous fluid due to a rotating disk. The disk is assumed to be rotating about the $z$-axis with a constant angular velocity $\Omega$ in the cylindrical coordinates $(r, \theta, z)$. The terminology is given for an external uniform magnetic field and electric field in Turkyilmazoglu [37] and Uygun [38] is followed. Respectively, Continuity equation, the Navier-Stokes equations including the Lorentz force and energy equation with viscous dissipation and Joule heating are given by,

$$
\begin{gathered}
\nabla \cdot \mathbf{v}=0 \\
\rho\left[\frac{\partial \mathbf{v}}{\partial t}+(\mathbf{v} \cdot \nabla) \mathbf{v}\right]=-\nabla p+\frac{1}{R e}\left[\nabla^{2} \mathbf{v}\right]+M_{n}(j \times B)_{i}, \\
\rho\left[\frac{\partial T}{\partial t} \quad+(\mathbf{v} \cdot \nabla) T\right]=M_{\infty}^{2}(\Gamma-1)\left[\frac{\partial p}{\partial t}+(\mathbf{v} \cdot \nabla) p\right]+\frac{1}{\operatorname{Pr}} \frac{1}{R e}\left[\nabla^{2} T\right] \\
+\frac{\Gamma-1}{\operatorname{Re}} M_{\infty}^{2}[\Phi]+M_{n}(\Gamma-1) M_{\infty}^{2} \frac{j^{2}}{\sigma} .
\end{gathered}
$$

The term $M_{n}(j \times B)_{i}$ in Eq.(2.2) represents Lorentz force terms. The last two terms in the right-hand-side of Eq.(2.3) represent

$$
\Phi=\left(\frac{\partial u}{\partial z}\right)^{2}+\left(\frac{\partial v}{\partial z}\right)^{2}
$$

the viscous dissipation and

$$
\frac{j^{2}}{\sigma}=\frac{1}{\left(1+m^{2}\right)^{2}}\left[\left(e_{r}+m u+v\right)^{2}+\left(m e_{r}-u+m v\right)^{2}+\left(1+m^{2}\right)^{2} e_{z}^{2}\right]
$$

Joule heating terms respectively.

The parameters in equations (2.1-2.3) are following: $\rho$ the density, $\mathbf{v}=(u, v, w)$ the velocity vector, $\nabla$ the usual gradient operator in cylindrical coordinates, $p$ the pressure, $R e$ is the Reynolds number characterizing the flow defined by $R e=\frac{s \Omega}{\nu}, \nu$ is the kinematic viscosity of the fluid, $M_{n}$ is the magnetic interaction parameter which represents the ratio between the magnetic force to the fluid interia force. The electric current is

$$
j=\sigma(e+\mathbf{v} \times B-\beta(j \times B)),
$$

where $j=\left(j_{r}, j_{\theta}, j_{z}\right)$ is the current density vector, $\sigma$ is the electrical conductivity, $e=$ $\left(e_{r}, e_{\theta}, e_{z}\right)$ is the electric field, $B$ is an external uniform magnetic field which has a constant magnetic flux density $B_{0}$, and the last term defines the Hall effect as $\beta$ is the Hall factor. Moreover, $\mathrm{T}$ is the temperature of the fluid, $\operatorname{Pr}=\frac{\mu c_{p}}{k}$ is the Prandatl number $c_{p}$ is the specific heat capacity, $\mu$ is the dynamical viscosity and $k$ is the thermal conductivity of the fluid, $\Gamma$ is the ratio of the specific heats, $M_{\infty}$ is the free-stream Mach number.

The basic flow of incompressible case, also called as Von Kármán's [20] flow will be considered here. Which means that the disk flow is assumed to evolve alongside the boundary layer coordinate $\eta=R e^{1 / 2} z$, in conformity with the self-similarity variables (see Hossain, Hossain\& Wilson [18]), 


$$
\begin{aligned}
& (u, v, w)=\left(r \Omega F(\eta), r \Omega G(\eta), R^{-1 / 2} H(\eta)\right), \\
& (p, T)=\left(\rho \Omega^{2} P(\eta), T_{\infty}+\left(T_{w}-T_{\infty}\right) \theta(\eta)\right),
\end{aligned}
$$

where $T_{w}$ is the temperature at the surface of the disk, $T_{\infty}$ is the temperature of the ambient fluid at a large distance from the disk. These quantities substitute into the governing equations (2.1-2.3), and also neglect terms of $O\left(R e^{-1}\right)$, the disk flow quantities are determined from the subsequent equations and boundary conditions appropriate to the stretchable disk flow as,

$$
\begin{array}{ll}
2 F \quad+H^{\prime}=0, \\
F^{2} \quad-G^{2}+F^{\prime} H-F^{\prime \prime}-\frac{M_{n}}{1+m^{2}}[-m \gamma-F+m G]+\gamma^{2}=0, \\
2 F G \quad+G^{\prime} H-G^{\prime \prime}-\frac{M_{n}}{1+m^{2}}[\gamma-G-m F]=0, \\
P^{\prime} \quad+H^{\prime} H-H^{\prime \prime}=0, \\
\frac{1}{P r} \theta^{\prime \prime} & -H \theta^{\prime}+E_{c}\left[\gamma^{2} F+F^{2}+G^{2}\right]+\frac{M_{n} E_{c}}{\left(1+m^{2}\right)^{2}}\left[(-\gamma+m F+G)^{2}\right. \\
& \left.+(-m \gamma-F+m G)^{2}\right]=0, \\
& F-1=G-\omega=H=\theta-1=0 \quad \text { at } \eta=0, \\
& F \rightarrow 0, \quad G \rightarrow \gamma, \theta \rightarrow 0, \quad \text { as } \eta \rightarrow \infty,
\end{array}
$$

where $E_{c}=\frac{M_{\infty}^{2}(\Gamma-1)}{T_{w}-T_{\infty}}$ is the Eckert number. $\omega=\Omega / s$ represents a rotation strength parameter measuring the ratio of swirl to stretch and $\omega=0$ means a pure stretching without rotation. $\gamma$ is the radial electric parameter caused by the electric field applied in a radial direction. Noting that a prime denotes derivative with respect to $\eta$. We should denote that equations (2.5) and boundary conditions (2.6) can be described as an extension of the problem of Fang [13] in the nonmagnetic and non-heat conducting flow case. It can be easily shown that the integration of the third momentum equation implements the stretching pressure.

The shear stress of fluid is directly proportional to the force applied and inversely proportional to its viscosity. This relationship is now known as Newton's Law of Viscosity. Therefore, applying the Newtonian formula, the radial component $\tau_{r}$ and tangential component $\tau_{\theta}$ of the shear stress are respectively obtained by

$$
\begin{aligned}
\tau_{r} & =\left(\frac{\partial u}{\partial z}\right)_{w}=r \Omega \sqrt{\frac{\Omega}{\nu}} F^{\prime}(0) \\
\tau_{\theta} & =\left(\frac{\partial v}{\partial z}\right)_{w}=r \Omega \sqrt{\frac{\Omega}{\nu}} G^{\prime}(0)
\end{aligned}
$$

Of physical interest is also the magnitude of the constant axial velocity at infinity, given by $H(\infty)$.

The heat transfer from the disk surface to the fluid is computed by the application of the Fourier's law, and using transformation for heat term we get,

$$
q=-k\left(\frac{\partial T}{\partial z}\right)_{w}=-k\left(T_{w}-T_{\infty}\right) \sqrt{\frac{\Omega}{\nu}} \frac{d \theta(0)}{d \eta},
$$

by rephrasing the heat transfer result in terms of the Nusselt number, defined as

$$
N_{u}=\frac{q \sqrt{\frac{\nu}{\Omega}}}{k\left(T_{w}-T_{\infty}\right)}
$$

Therefore the equation (2.9) becomes,

$$
N_{u}=-\frac{d \theta(0)}{d \eta}
$$


We here prefer, due primarily to its high accuracy, the use of spectral collocation method incorporating Chebyshev polynomials. Having mapped the physical semi-infinite region onto the computational domain via a suitable transformation, a staggered mesh is then chosen so that Chebyshev Collocation points are interchanged between the Gauss-Lobatto points and Gauss points, whenever necessary, particularly to deal with the pressure perturbation at the wall. As a result of the spectral discretization, the system together with the relevant initial and boundary conditions were put into a large matrix form and then solved using LU matrix decomposition combined with a usual Newton-Raphson search procedure.

In this study, stated above a matrix method, which is called the Chebyshev-matrix method, for the approximate solution of linear differential equations in term of Chebyshev collocations is presented for numerical solution of the equations (2.5) under the initial and boundary conditions (2.6). The method is based on the truncated Chebyshev series of the functions in the equations and then substituting their matrix forms into the given equations. Using the Chebyshev collocation points, this method transforms the differential-integral equations to a matrix equation which corresponds to a system of linear algebraic equations with unknown Chebyshev coefficients. Therefore this allows us to make use of the computer for the solution of the equations. Also, the Chebyshev collocation method can be used for differential and integral equations.

\section{Results and discussions}

In the literature, many researchers have applied different methods to solve the system of the equations. Some of these methods they use to reach their results can be given as: a finite-difference method, a special technique, and also Chebyshev collocation method. For instance, Attia [4], Jasmine \& Gajjar [19], Sahoo [27], Turkyilmazoglu [33,36] and Uygun $[38,39]$ achieved their works using these technics.

Here we prefer the use of the Spectral Collocation method. In the paper we numerically solved the system of differential equations (2.5) together with the relevant boundary conditions (2.6). The numerical results are obtained by utilizing the Spectral Chebyshev collocation scheme basing on Chebyshev polynomials. We briefly summarize the numerical scheme as follows: Initially, nonlinear terms are linearized with the Newton linearization technique in the given equations. After that, using the Chebyshev collocation points, the linearized equations are transformed to a matrix equation with unknown Chebyshev coefficients and then the matrix system is solved by decomposition technique.

\begin{tabular}{|c|rc|cc|}
\hline$M_{n}$ & \multicolumn{2}{|c|}{$F^{\prime}(0)$} & \multicolumn{2}{c|}{$-G^{\prime}(0)$} \\
\hline \multirow{3}{*}{0.0} & Present & Sahoo & Present & Sahoo \\
\cline { 2 - 4 } & 0.510232 & 0.510214 & 0.615922 & 0.615909 \\
\hline
\end{tabular}

Table 1. Comparison of the numerical solutions of shear stress coefficients in radial and tangential directions $F^{\prime}(0),-G^{\prime}(0)$ respectively.

\begin{tabular}{|r|c|c|cc|lr|}
\hline$M_{n}$ & $P_{r}$ & $\Gamma$ & \multicolumn{2}{|c|}{$H(\infty)$} & \multicolumn{2}{|c|}{$-\theta^{\prime}(0)$} \\
\hline & & & Present & Turkyilmazoglu & Present & Turkyilmazoglu \\
\cline { 4 - 7 } 0.5 & \multirow{2}{*}{0.0} & -0.458880064 & -0.45888005 & 0.282655934 & 0.28265593 \\
\hline
\end{tabular}

Table 2. Comparison of numerical solutions of the vertical velocity, $H(\infty)$ and coefficients of the heat transfer, $-\theta^{\prime}(0)$. 
To verify the accuracy of the numerical scheme, as well as, to validate the code, we compared our results with the outcome of the studies by Sahoo [27] and Turkyilmazoglu [33]. For comparison purpose, the results of Sahoo [27], and Turkyilmazoglu [33] are tabulated in Table 1 and Table 2, which presents a clear evidence for accuracy of the numerical method.

\subsection{Outcomes and debates of graphs}

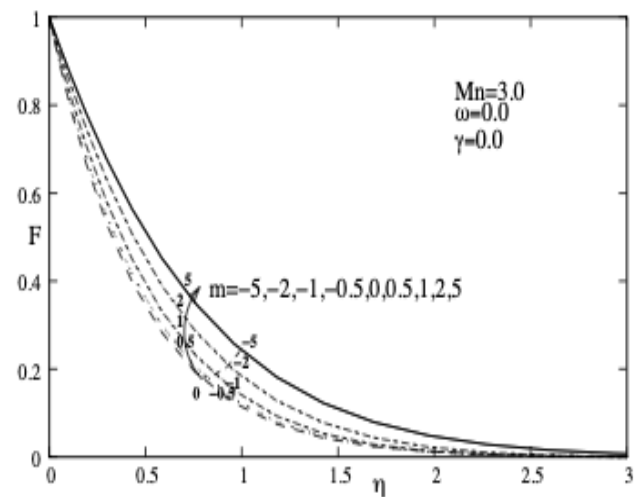

(a)

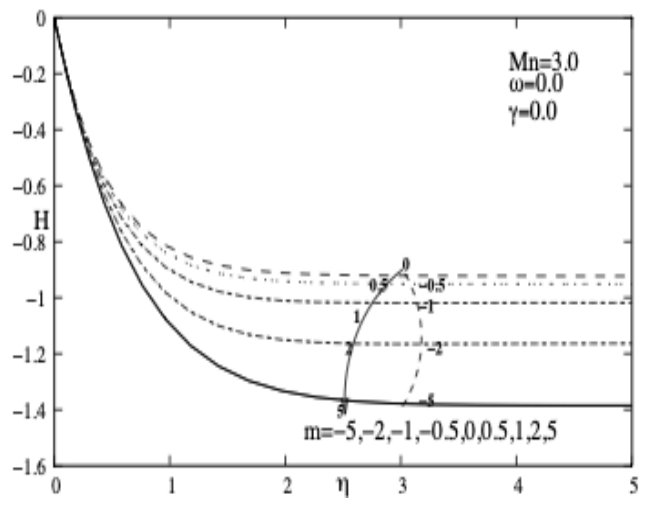

(c)

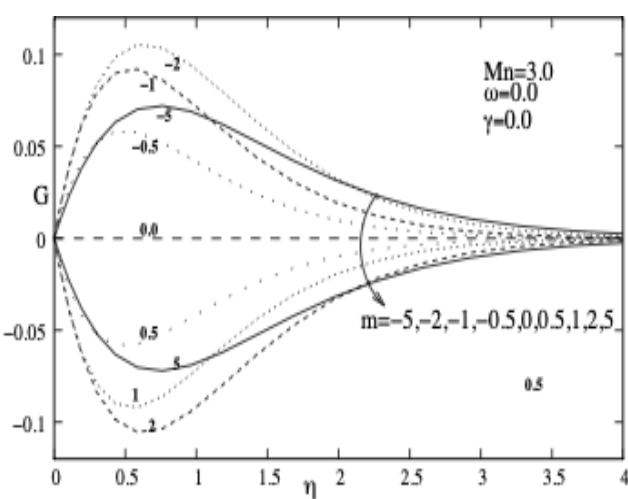

(b)

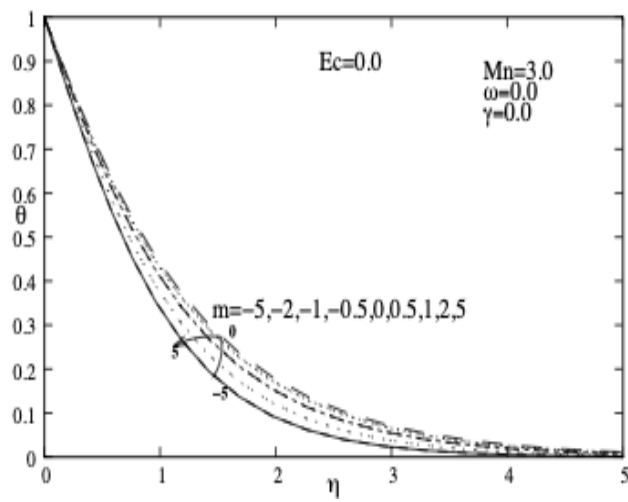

(d)

Figure 1. Effects of Hall parameter $m$ are demostrated on the flow quantities for the selected paremeters $M_{n}=3.0, \gamma=0.0, \omega=0.0, \operatorname{Pr}=1.0$ and $E_{c}=0.0$, respectively in (a) for radial velocity profiles $F$, in (b) for tangential velocity profiles $G$, in (c) for axial velocity profiles $H$ and in (d) for temperature profiles $\theta$.

The importance of the Hall effect is supported by the need to determine accurately carrier density, electrical resistivity, and the mobility of carriers in semiconductors. The Hall effect provides a relatively simple method for doing this. Because of its simplicity, low cost, and fast turnaround time, it is an indispensable characterization technique in the semiconductor industry and in research laboratories. Furthermore, two Nobel prizes $(1985,1998)$ are based upon the Hall effect.

The Hall effect is the production of a voltage difference across an electrical conductor, transverse to an electric current in the conductor and to an applied magnetic field perpendicular to the current. It was discovered by Edwin Hall [16] in 1879.

The Hall effect is due to the nature of the current in a conductor. Current consists of the movement of many small charge carriers, typically electrons, holes, ions or all three. 


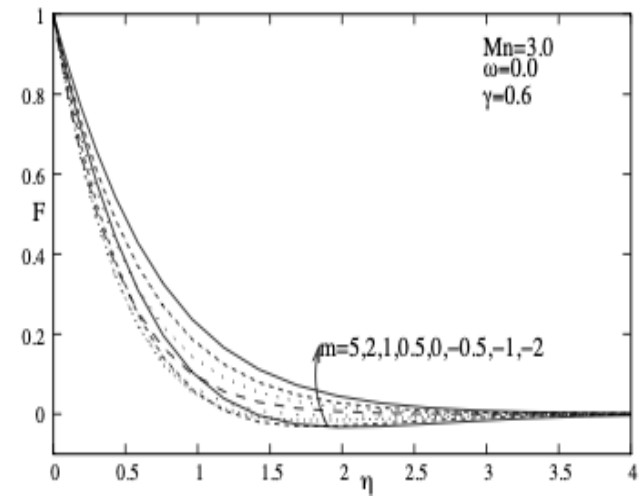

(a)

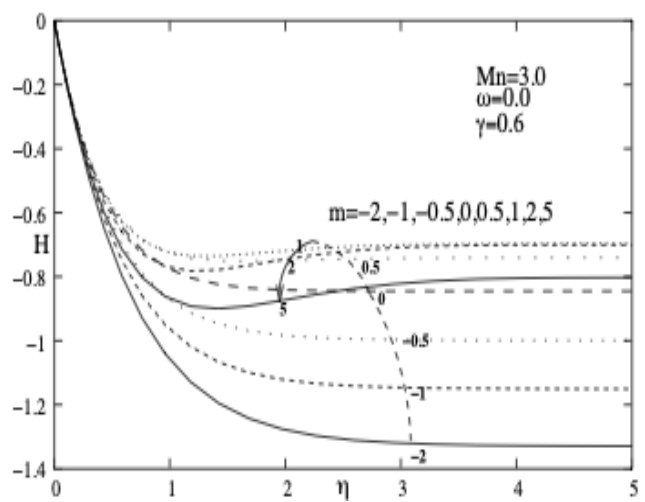

(c)

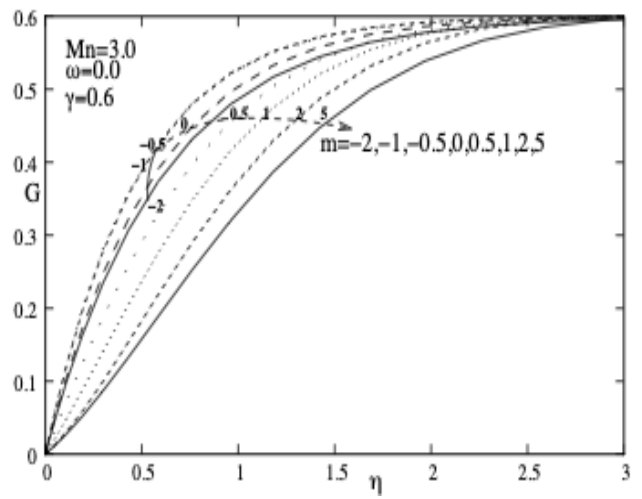

(b)

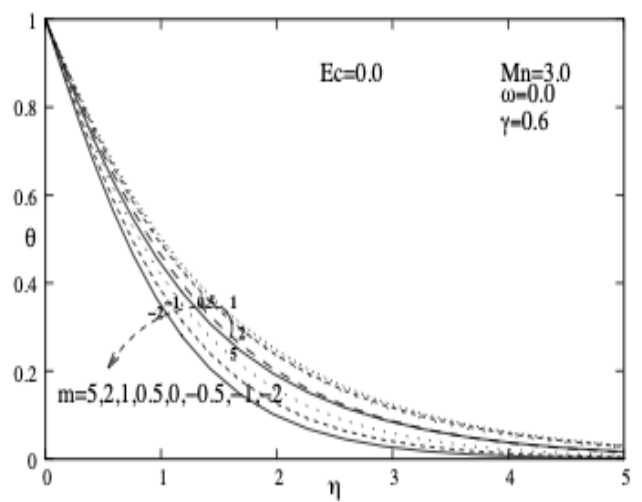

(d)

Figure 2. Effects of Hall parameter $m$ are demostrated on the flow quantities for the selected paremeters $M_{n}=3.0, \gamma=0.6, \omega=0.0, \operatorname{Pr}=1.0$ and $E_{c}=0.0$, respectively in (a) for radial velocity profiles $F$, in (b) for tangential velocity profiles $G$, in (c) for axial velocity profiles $H$ and in (d) for temperature profiles $\theta$.

When a magnetic field is present, these charges experience a force, called the Lorentz force. When such a magnetic field is absent, the charges follow approximately straight, 'line of sight' paths between collisions with impurities, phonons, etc. However, when a magnetic field with a perpendicular component is applied, their paths between collisions are curved, thus moving charges accumulate on one face of the material. This leaves equal and opposite charges exposed on the other face, where there is a scarcity of mobile charges. The result is an asymmetric distribution of charge density across the Hall element, arising from a force that is perpendicular to both the 'line of sight' path and the applied magnetic field. The separation of charge establishes an electric field that opposes the migration of further charge, so a steady electric potential is established for as long as the charge is flowing. As a result, the Hall effect is very useful as a means to measure the magnetic field.

Physically, when the Hall parameter is low, the trajectories of electrons between two encounters with heavy particles (neutral or ion) are almost linear. But if the Hall parameter is high, the electron movements are highly curved. The current density vector is no more colinear with the electric field vector. It means that, the Hall parameter value increases with the magnetic field strength.

The Hall parameter $m$ appears in the magnetic force terms of continuity equations and in Joule heating term of energy equation. Considering these terms, we conclude that for 


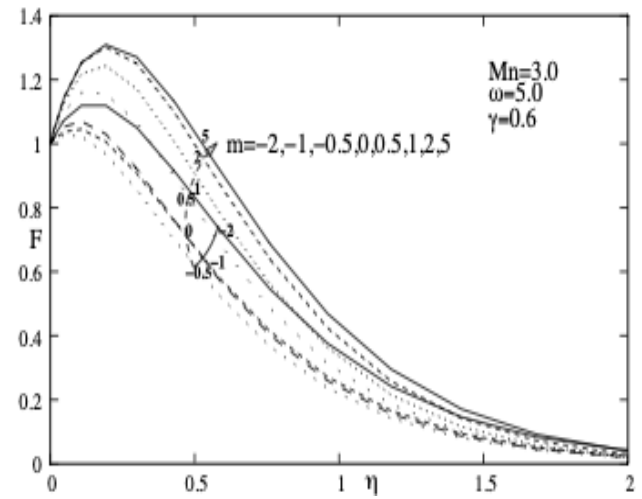

(a)

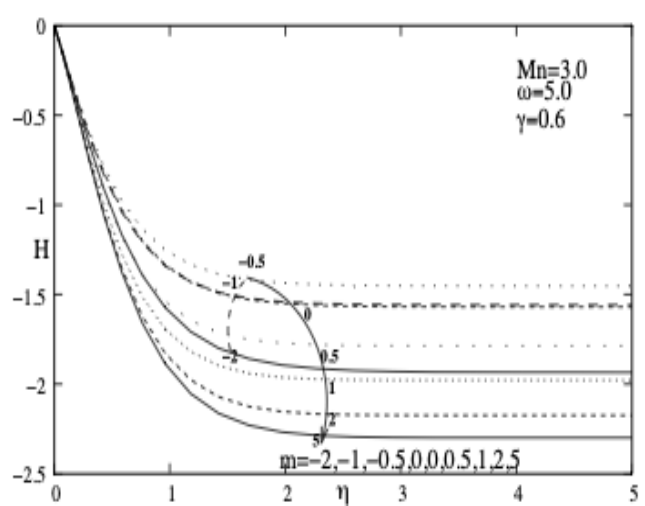

(c)

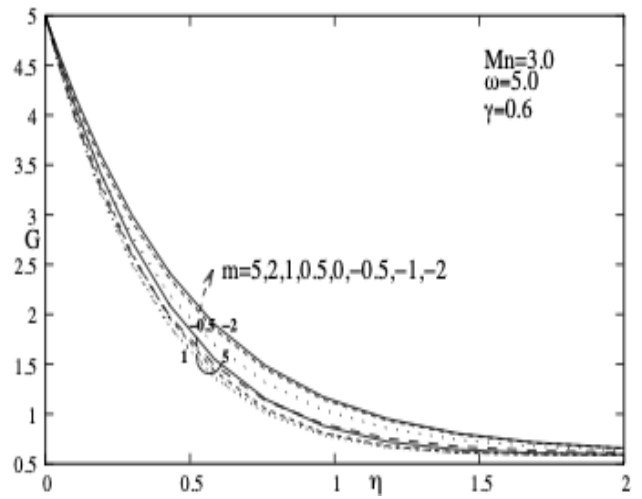

(b)

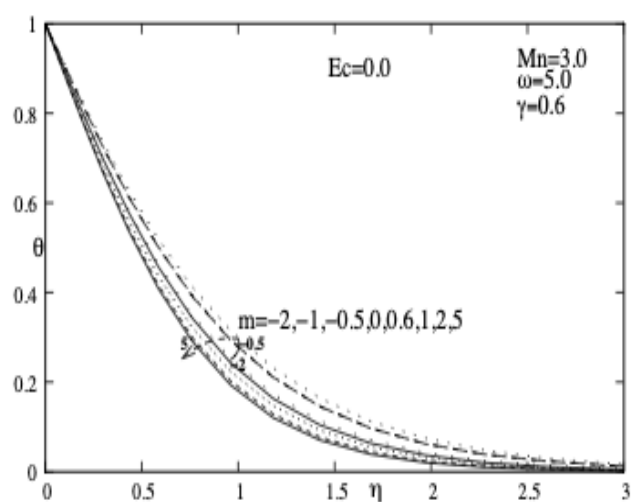

(d)

Figure 3. Effects of Hall parameter $m$ are demostrated on the flow quantities for the selected paremeters $M_{n}=3.0, \gamma=0.6, \omega=5.0, \operatorname{Pr}=1.0$ and $E_{c}=0.0$, respectively in (a) for radial velocity profiles $F$, in (b) for tangential velocity profiles $G$, in (c) for axial velocity profiles $H$ and in (d) for temperature profiles $\theta$.

varying values of Hall parameter $m$, the impact of Hall parameter $m$ on the numerator is stronger than its impact on the denominator.

Equations (2.5) under the conditions (2.6) are solved to compute the various velocity profiles and temprature profiles as depicted in Figures (1-7). These graphs show effects of the various Hall parameters $m$ for the two different rotation numbers $\omega=0.0, \omega=5.0$, the three different radial electric parameters $\gamma=-0.5, \gamma=0.0, \gamma=0.6$, and Eckert numbers $E_{c}=-2.0, E_{c}=0.0, E_{c}=2.0$ respectively, fixed Magnetic interaction number $M_{n}=3.0$, and fixed Prandtl number $P_{r}=1.0$.

In the event that the radial electric parameter doesn't exist (when $\gamma=0.0$ ) and without rotation $(\omega=0.0)$, all the velocity profiles and the temperature profile behave like a torque when the Hall parameter $m$ varies from the biggest value to the smallest value or vice versa, as shown in Figures (1(a)-1(d)). Also, these graphs demonstrate that the tangential velocity profiles get symmetric values, although the radial velocity $F$, the axial velocity profiles $H$, and the temperature profiles $\theta$ take the same values at the several symmetric Hall parameters $m$ for fixed Magnetic interaction number $M_{n}=3.0$, Eckert number $E_{c}=0.0$, and Prandtl number $P_{r}=1.0$. That is to say, while acting as an odd function in the profiles of axial velocity $G$, radial velocity $F$, axial velocity $H$, and temperature profiles $\theta$ behave like an even function at the symmetric Hall parameter $m$ values. 


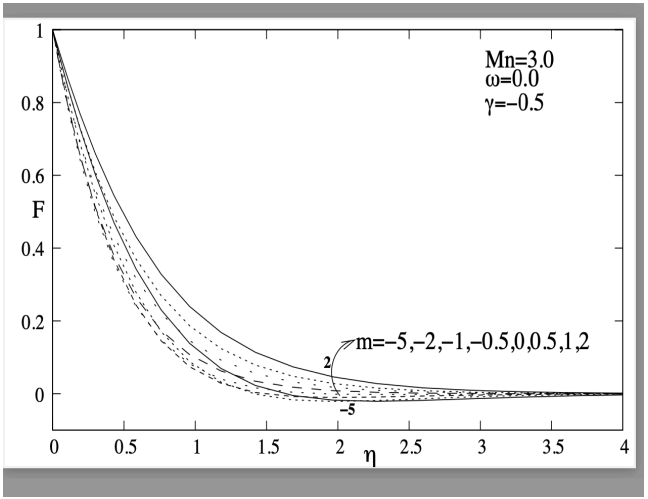

(a)

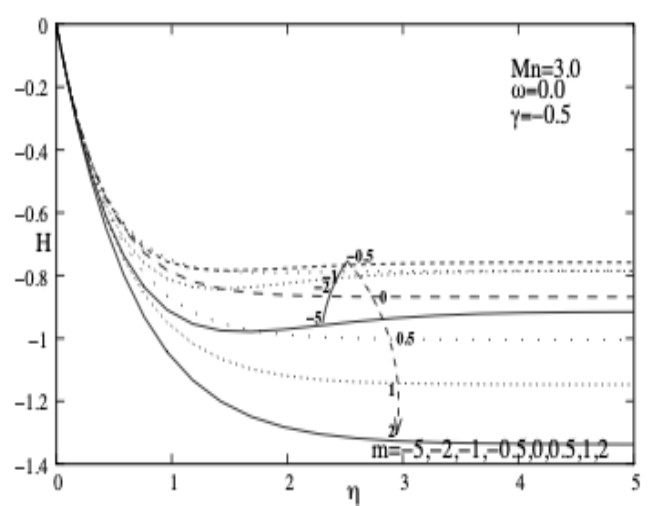

(c)

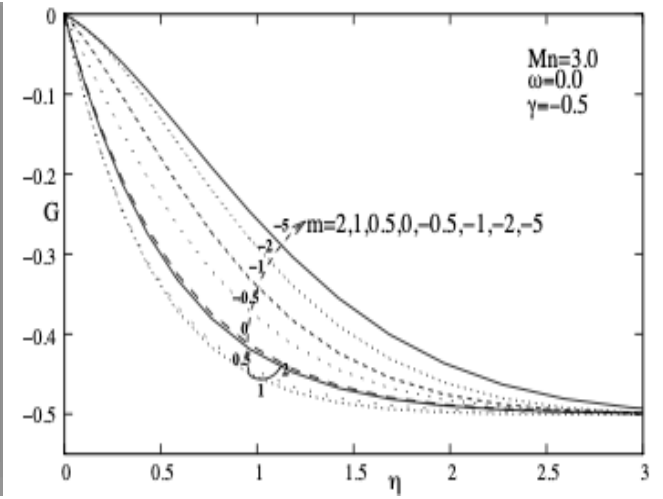

(b)

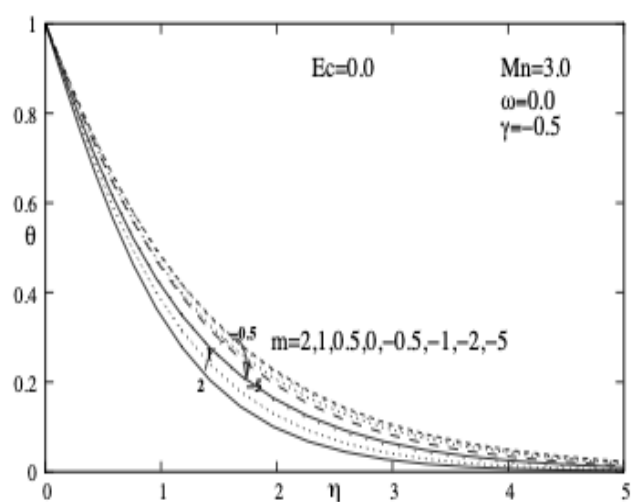

(d)

Figure 4. Effects of Hall parameter $m$ are demostrated on the flow quantities for the selected paremeters $M_{n}=3.0, \gamma=-0.5, \omega=0.0, \operatorname{Pr}=1.0$ and $E_{c}=0.0$, respectively in (a) for radial velocity profiles $F$, in (b) for tangential velocity profiles $G$, in (c) for axial velocity profiles $H$ and in (d) for temperature profiles $\theta$.

In case of becoming positive $\gamma=0.6$ or negative $\gamma=-0.5$ values of the radial electric parameter, it is observed that when the Hall parameter $m$ decreases from the biggest value to the smallest value or vice versa, all components of the velocity profiles, and also temperature profiles forms similar to a saddle shape except for temperature profile at rotation number $\omega=5.0$ in Figure 5(d). All of these relations can be fairly seen in figures (2-5) for fixed Magnetic interaction number $M_{n}=3.0$, Eckert number $E_{c}=0.0$, and Prandtl number $P_{r}=1.0$.

It should be noted that, in both cases for without rotation, the size of the interval of $\eta$ first shrinks and then expands at the radial velocity $F$, and the tangential velocity profiles $G$, while Hall parameter $m$ is monotone, that is either decreasing or increasing. There is a similar exchange in the temperature profiles $\theta$, however the situation is exactly reversed. According to graphs (2) and (4), there is no meaningful change in radial and tangential component of the velocity profiles and also temperature profiles when the Hall parameter varies from the smallest value to the biggest value or vice versa. Meanwhile, on the axial component of the velocity can be visualized as in graph 2(c) and in graph 4(c). These graphs demonstrate that the increment or descent in the Hall parameter causes first increment and then descent in the axial velocity values for both positive $\gamma=0.6$ and negative $\gamma=-0.5$ electric parameters in radial direction. 


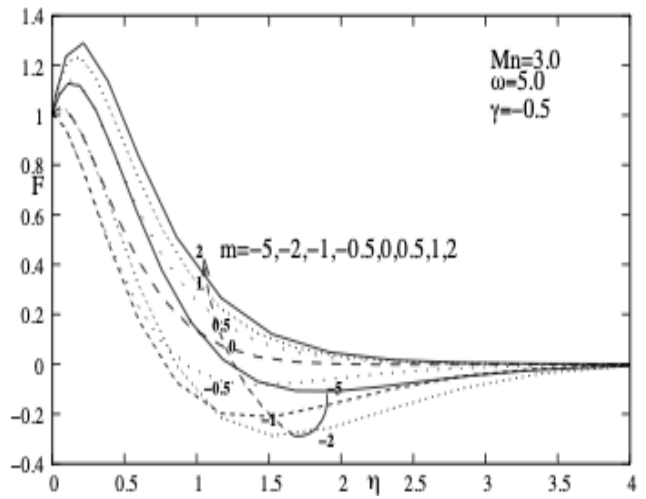

(a)

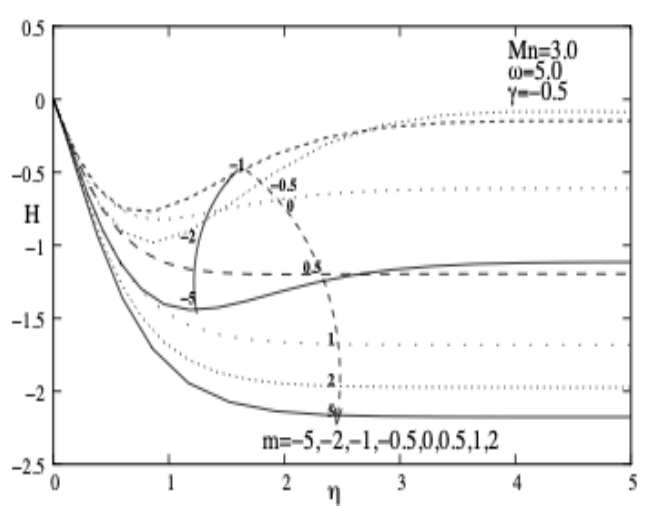

(c)

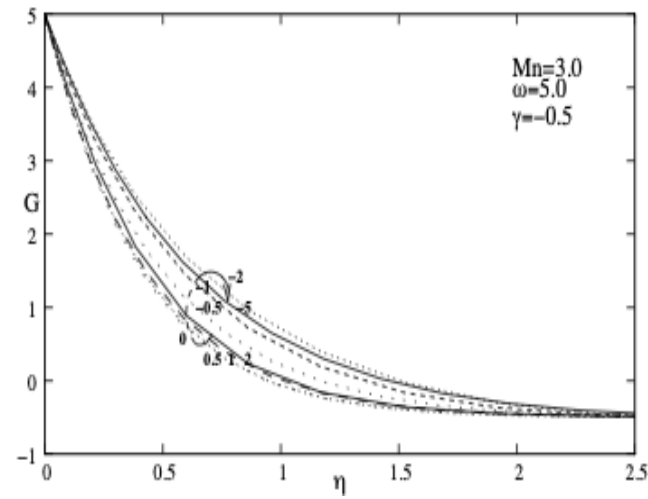

(b)

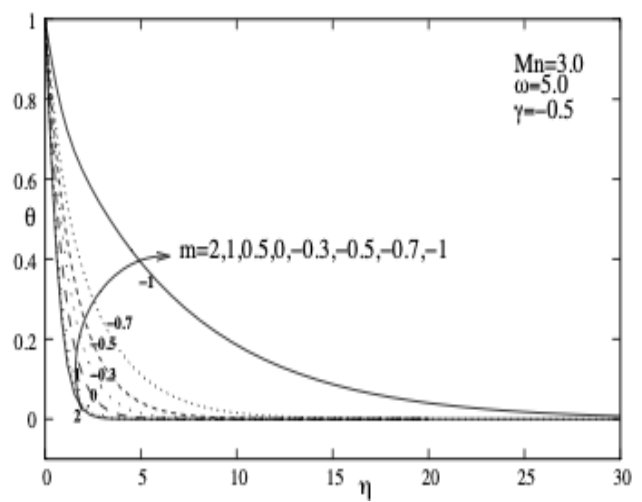

(d)

Figure 5. Effects of Hall parameter $m$ are demostrated on the flow quantities for the selected paremeters $M_{n}=3.0, \gamma=-0.5, \omega=5.0, \operatorname{Pr}=1.0$ and $E_{c}=0.0$, respectively in (a) for radial velocity profiles $F$, in (b) for tangential velocity profiles $G$, in (c) for axial velocity profiles $H$ and in (d) for temperature profiles $\theta$.

In consequence of getting the value 5.0 of the rotation parameter, when the electric parameter on the radial direction has a positive value $(\gamma=0.6)$, a similar effect is observed on the all components of the velocity profiles and also the temperature profiles as illustrated in figures (3(a)-(d)) for changing Hall parameters. In other words, all these profiles almost identical with the previous one, therefore, it is similar to the shape of saddle as Hall parameter increases or decreases.

Moreover, in cases where the electrical parameter has positive $\gamma=0.6$ and negative $\gamma=-0.5$ values, all components of the velocity profiles are similar to each other. The impacts of the negative Hall parameters on the these profiles can be clearly seen in graphs (5(a)-(c)) when the radial electric parameter takes negative value $\gamma=-0.5$. These graphs delineate that the negative Hall parameter has a prominent effect on especially radial and axial components of velocity. Also, the impacts of Hall numbers on temperature profiles are depicted in graph 1(d). However, these show that the effects of the negative Hall parameters on the all velocity profiles are very different from on the temperature profiles. In current state can be explained that the size of the interval of $\eta$ increases while a Hall parameter decreases, especially in negative Hall parameters.

Finally, let us examine the behavior of the temperature profiles for two different Eckert numbers $E_{c}=-2.0$ and $E_{c}=2.0$ in two cases. These cases in which a rotation parameter becomes $\omega=0.0$ and it gets the value $\omega=5.0$. In the first case, while temperature profile 


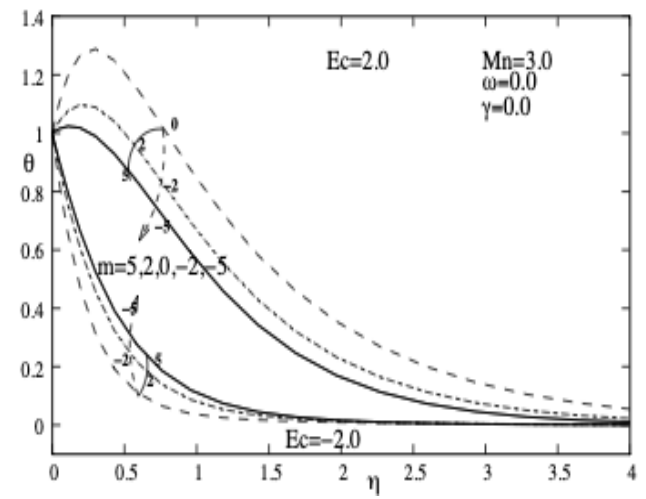

(a)

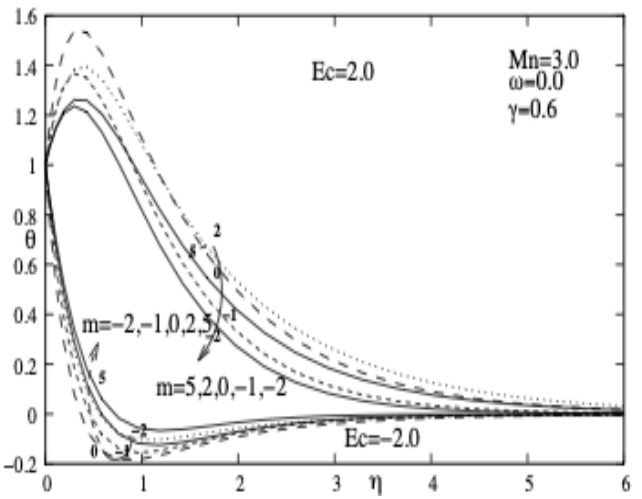

(b)

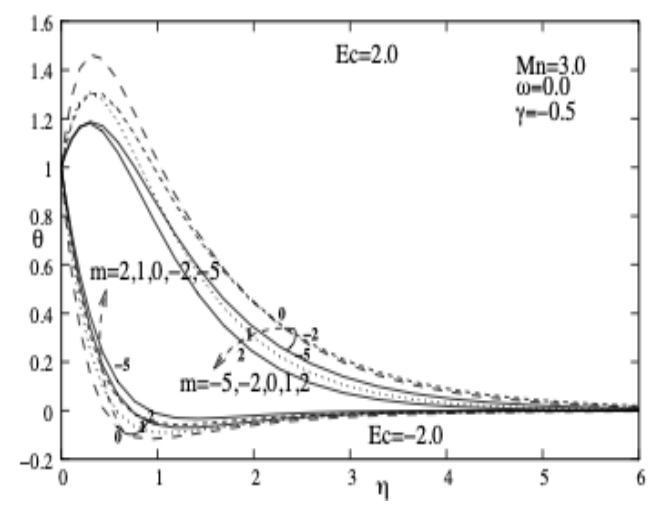

(c)

Figure 6. Effects of Hall parameter $m$ are demostrated on the temperature profiles $\theta$ for the selected paremeters $M_{n}=3.0, \omega=0.0, P_{r}=1.0$ for two different Eckert numbers $E_{c}=2.0$ and $E_{c}=-2.0$, and for three different radial electric parameters, respectively in (a) $\gamma=0.0$, in (b) $\gamma=0.6$, and in (c) $\gamma=-0.5$.

behaves like a torque for radial electric parameter $\gamma=0.0$, but it forms a saddle shape in case of having positive or negative values of the electric parameter in the radial direction then this seems to occur for Eckert number $E c=0.0$. In the second case, temperature profile shows similar behavior to the previous case for the radial electric parameters $\gamma=$ $0.0, \gamma=0.6$. On the other hand, at the negative radial electric parameter $\gamma=-0.5$, due to decreasing Hall parameter, the size of the interval of $\eta$ expands, furthermore, particularly in negative Hall parameters, the temperature profiles gradually grow or shrink for Eckert number $E_{c}=2.0$ or $E_{c}=-2.0$ respectively. The impact of the Hall parameter on temperature profiles is emphasized in Figures (6-7) for fixed magnetic interaction number $M_{n}=3.0$, and for fixed Prandtl number $P_{r}=1.0$.

\subsection{Outcomes and debates of tables}

The history of the Hall effect begins in 1879 when Edwin H. Hall discovered that a small transverse voltage appeared across a current-carrying thin metal strip in an applied magnetic field. The basic physical principle underlying the Hall effect is the Lorentz force, which is a combination of two separate forces: the electric force and the magnetic force.

Physically, the trajectories of electrons are curved by the Lorentz force. Nevertheless, when the Hall parameter is low, their motion between two encounters with heavy particles (neutral or ion) is almost linear. But if the Hall parameter is high, the electron movements 


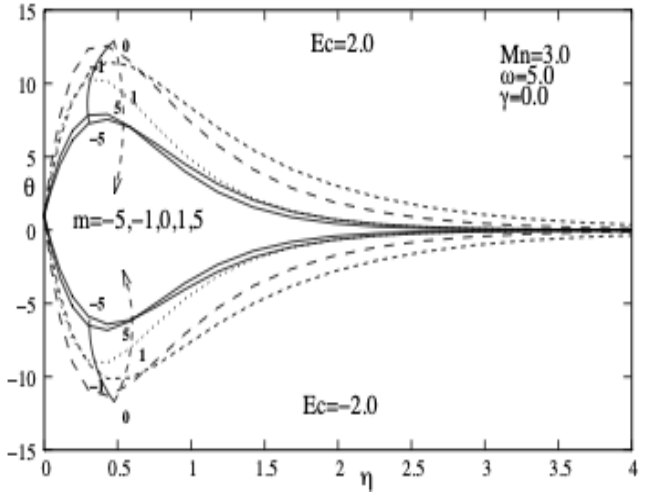

(a)

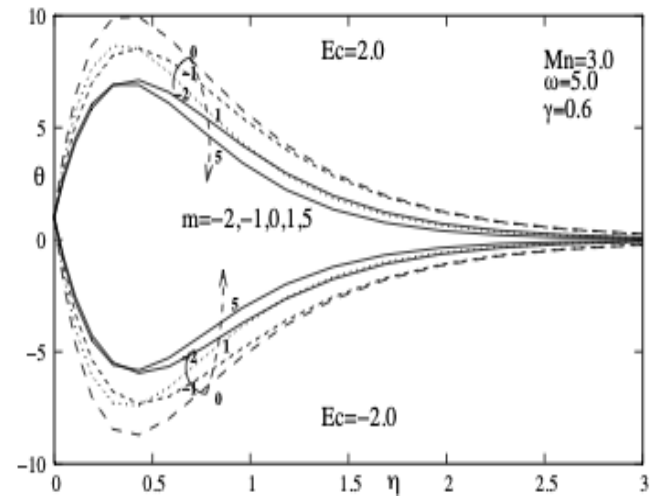

(b)

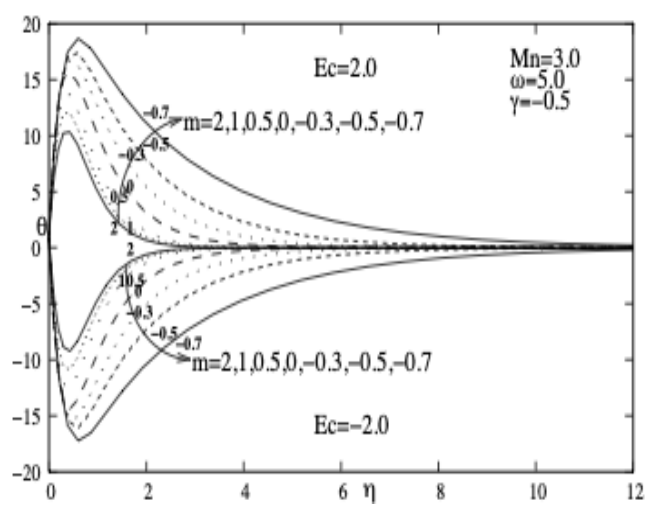

(c)

Figure 7. Effects of Hall parameter $m$ are demostrated on the temperature profiles $\theta$ for the selected paremeters $M_{n}=3.0, \omega=5.0, P_{r}=1.0$ for two different Eckert numbers $E_{c}=2.0$ and $E_{c}=-2.0$, and for three different radial electric parameters, respectively in (a) $\gamma=0.0$, in (b) $\gamma=0.6$, and in (c) $\gamma=-0.5$.

are highly curved. The current density vector is no longer collinear with the electric field vector.

In this work, variations of the radial shear stress $F^{\prime}(0)$, tangential shear stress $G^{\prime}(0)$, the velocity in the radial direction $H(\infty)$ and coefficients of heat transfer $-\theta^{\prime}(0)$ have been tabulated for various Hall parameter $m$ for the fixed magnetic interaction number $M_{n}=3.0$, for the two different rotation parameters $\omega=0.0, \omega=5.0$, for three different radial electric parameters $\gamma=-0.5, \gamma=-0.0, \gamma=0.6$ and for the three different Eckert numbers $E_{c}=-2.0, E_{c}=0.0, E_{c}=2.0$ respectively, illustrated in Tables (3-6).

When the positive Hall parameter $m$ increases, the radial shear stress increases for the all electric fields with or without rotation, however, there is a reverse effect at the negative Hall numbers, as seen in tables (3) and (5).

The tangential shear stress decreases when the negative Hall parameter increases, but it increases as the positive Hall parameter increases at the negative radial field for with or without rotation. In the case of without rotation, the increasing Hall parameter causes decreases in the tangential shear stress for having the value $\gamma=0.0$ of radial electric parameter, and it increases when the negative Hall parameter increases, as it decreases if the positive Hall parameter increases at the positive radial field. Both at the taking zero value of the radial electric parameter and at the positive radial field for rotation case, the tangential shear stress decreases/increases when the negative/positive Hall parameter increases. All these relations can be concluded from tables (3) and (5). 


\begin{tabular}{|c|c|c|c|c|c|}
\hline$\omega$ & $\gamma$ & $m$ & $F^{\prime}(0)$ & $G^{\prime}(0)$ & $H(\infty)$ \\
\hline \multirow{15}{*}{0.0} & \multirow{5}{*}{-0.5} & -2.0 & -1.854945 & 0.118076 & 0.785723 \\
\hline & & -0.5 & -2.232868 & -0.509406 & -0.784548 \\
\hline & & 0.0 & -2.172160 & -0.878613 & -0.868367 \\
\hline & & 0.5 & -1.873577 & -1.110068 & -1.004486 \\
\hline & & 2.0 & -1.323200 & -0.900473 & -1.337277 \\
\hline & \multirow{5}{*}{0.0} & -2.0 & -1.467409 & 0.430753 & -1.163193 \\
\hline & & -0.5 & -1.962015 & 0.314871 & -0.951425 \\
\hline & & 0.0 & -2.084846 & 4.69E-014 & -0.922605 \\
\hline & & 0.5 & -1.962015 & -0.314871 & -0.951425 \\
\hline & & 2.0 & -1.467409 & -0.430753 & -1.163193 \\
\hline & \multirow{5}{*}{0.6} & -2.0 & -1.327327 & 0.987694 & -1.329457 \\
\hline & & -0.5 & -1.878534 & 1.267034 & -0.998957 \\
\hline & & 0.0 & -2.210470 & 1.055630 & -0.844945 \\
\hline & & 0.5 & -2.307 & 0.678969 & -0.740608 \\
\hline & & 2.0 & -1.957950 & 0.238829 & -0.700847 \\
\hline
\end{tabular}

Table 3. Shear stress coefficients $F^{\prime}(0)$ and $G^{\prime}(0)$, vertical velocity $H(\infty)$ are tabulated at some chosen Hall parameters $m$, for the three different radial electric parameters $\gamma=-0.5, \gamma=0.0$ and $\gamma=0.6$ respectively, for fixed Magnetic interaction number $M_{n}=3.0$ and for fixed rotation number $\omega=0.0$.

\begin{tabular}{|c|c|c|c|c|c|c|c|}
\hline$\omega$ & $E_{c}$ & $\gamma$ & $m=-2.0$ & $m=-0.5$ & $m=0.0$ & $m=0.5$ & $m=2.0$ \\
\hline \multirow{9}{*}{0.0} & \multirow{3}{*}{-2.0} & -0.5 & 3.395840 & 4.627154 & 4.888683 & 4.502633 & 3.102112 \\
\hline & & 0.0 & 2.549249 & 3.618659 & 3.879251 & 3.618659 & 2.549249 \\
\hline & & 0.6 & 3.374241 & 4.902956 & 5.332295 & 5.058447 & 3.736165 \\
\hline & \multirow{3}{*}{0.0} & -0.5 & 0.659184 & 0.630868 & 0.660975 & 0.714658 & 0.816639 \\
\hline & & 0.0 & 0.778826 & 0.696492 & 0.681802 & 0.696492 & 0.778826 \\
\hline & & 0.6 & 0.814909 & 0.712799 & 0.651599 & 0.611263 & 0.622617 \\
\hline & \multirow{3}{*}{2.0} & -0.5 & -2.077471 & -3.365416 & -3.566732 & -3.073316 & -1.468832 \\
\hline & & 0.0 & -0.991597 & -2.225673 & -2.515646 & -2.225673 & -0.991597 \\
\hline & & 0.6 & -1.744422 & -3.477358 & -4.029096 & -3.835920 & -2.490931 \\
\hline
\end{tabular}

Table 4. Heat transfer parameter $-\theta^{\prime}(0)$ is tabulated at some chosen Hall parameters $m$ for the three different radial electric parameters $\gamma=-0.5, \gamma=0.0$, $\gamma=0.6$, and Eckert numbers $E_{c}=-2.0, E_{c}=0.0, E_{c}=2.0$ respectively, fixed Magnetic interaction number $M_{n}=3.0$, fixed rotation number $\omega=0.0$, and fixed Prandtl number $P_{r}=1.0$.

The vertical velocity increases/decreases at the infinity when the negative/ positive Hall parameter increases for the negative $(\gamma=-0.5)$ radial electric parameter and also having zero value $(\gamma=0.0)$ of radial electric parameter at the far from the disk. It increases in case of having positive electric parameters in the radial direction if there is increament of the Hall parameter. All of these behaviors of the vertical velocity are decuded from table (3) in the nonrotation disk. Also, table (5) demonstrates that the vertical velocity decreases far from the rotation disk for the increasing Hall parameter at the negative electric field in the radial direction. The increament in the negative/positive Hall parameter causes increases/decreases in the vertical velocity at the infinity for both becoming zero or positive value of the radial electric parameter in the rotation disk.

The impact of Hall numbers on heat tranfer parameter can be deduced from tables (4) and (6). Regardless of nonrotation or rotation disk, it can be seen that the heat tranfer 


\begin{tabular}{|c|c|c|c|c|c|}
\hline$\omega$ & $\gamma$ & $m$ & $F^{\prime}(0)$ & $G^{\prime}(0)$ & $H(\infty)$ \\
\hline \multirow{6}{*}{5.0} & & -2.0 & 0.899717 & -8.583750 & $-9.42 \mathrm{E}-002$ \\
& & -0.5 & $-6.16 \mathrm{E}-002$ & -11.252060 & -0.609877 \\
& -0.5 & 0.0 & 1.035168 & -12.763429 & -1.195614 \\
& & 0.5 & 2.557373 & -12.976875 & -1.682194 \\
\cline { 3 - 6 } & & 2.0 & 3.709262 & -11.229947 & -2.176914 \\
\cline { 3 - 6 } & & -2.0 & 1.534930 & -8.658559 & -1.397829 \\
& & -0.5 & 0.502729 & -10.710837 & -1.118283 \\
& & 0.0 & 1.370233 & -12.057284 & -1.429702 \\
& & 0.5 & 2.700050 & -12.305152 & -1.783035 \\
& & 2.0 & 3.785244 & -10.854629 & -2.246936 \\
\hline \multirow{5}{*}{0.6} & -2.0 & 1.923264 & -8.416167 & -1.934672 \\
& & -0.5 & 0.912643 & -9.970062 & -1.452679 \\
& & 0.0 & 1.554997 & -11.131026 & -1.558208 \\
& & 0.5 & 2.671395 & -11.403082 & -1.786789 \\
& & 2.0 & 3.663121 & -10.251231 & -2.173806 \\
\hline
\end{tabular}

Table 5. Shear stress coefficients $F^{\prime}(0)$ and $G^{\prime}(0)$, vertical velocity $H(\infty)$ are tabulated at some chosen Hall parameters $m$, for the three different radial electric parameters $\gamma=-0.5, \gamma=0.0$ and $\gamma=0.6$ respectively, for fixed Magnetic interaction number $M_{n}=3.0$ and for fixed rotation number $\omega=5.0$.

\begin{tabular}{|c|c|c|c|c|c|c|c|}
\hline$\omega$ & $E_{c}$ & $\gamma$ & $m=-2.0$ & $m=-0.5$ & $m=0.0$ & $m=0.5$ & $m=2.0$ \\
\hline \multirow{6}{*}{5.0} & & -0.5 & 68.271820 & 94.471808 & 102.204217 & 95.645113 & 67.677868 \\
& \multirow{4}{*}{2.0} & 0.0 & 47.849021 & 76.417962 & 85.863844 & 82.030346 & 60.471686 \\
\cline { 3 - 8 } & & 0.6 & 41.350049 & 61.312814 & 69.727930 & 67.878672 & 52.474675 \\
\cline { 2 - 8 } & & -0.5 & 0.310365 & 0.628128 & 0.847566 & 1.002861 & 1.127978 \\
& & 0.0 & 0.952788 & 0.820072 & 0.915726 & 1.028868 & 1.142697 \\
\cline { 2 - 8 } & \multirow{2}{*}{2.0} & -0.5 & -67.041602 & 0.912599 & 0.952620 & 1.033550 & 1.134726 \\
\hline & & 0.0 & -45.943444 & -74.777817 & -84.032391 & -79.972608 & -58.186292 \\
& & 0.6 & -39.266844 & -59.487614 & -67.822689 & -65.811572 & -50.205222 \\
\hline
\end{tabular}

Table 6. Heat transfer parameter $-\theta^{\prime}(0)$ is tabulated at some chosen Hall parameters $m$ for the three different radial electric parameters $\gamma=-0.5, \gamma=0.0$, $\gamma=0.6$, and Eckert numbers $E_{c}=-2.0, E_{c}=0.0, E_{c}=2.0$ respectively, fixed Magnetic interaction number $M_{n}=3.0$, fixed rotation number $\omega=5.0$, and fixed Prandtl number $P_{r}=1.0$.

parameter increases/decreases in the case of increasing negative/positive Hall parameter values for negative Eckert number $E_{c}=-2.0$. Besides this, if the Hall parameter gets opposite values, the effect on it becomes reversed for positive Eckert number $E_{c}=2.0$. These conclusions valid again for with or without rotation.

\section{Conclusions}

The velocity and temperature profiles governing the steady-incompressible boundary layer flow over a stretching disk have been obtained using self-consistent assumptions. The reconstructing equations have then been solved numerically by using Chebyshev collocation method. Then the behaviors of the velocity and temperature profiles are obtained and displayed graphically. 
The effects of Hall parameter are tabulated for the three different radial electric parameters, for three different Eckert numbers, for two different rotation parameters, and a fixed magnetic interaction parameter. One of the main outcomes of the present study is defining the effect of the Hall parameters on velocity and temperature profiles for varying Hall numbers. In case of decreasing values of Hall parameter with rotation on the negative radial electric field, size of the interval of $\eta$ expands in the temperature profiles, moreover, temperature profiles gradually grow/shrink for positive/negative Eckert numbers.

Acknowledgment. I would like to express my deep appreciation for the referees valuable contribution through their corrections and comments.

\section{References}

[1] L. Aisling and R. Ake, Crystal Growth of Single Salicylamide Crystals, Cryst Growth Des., 2019.

[2] T. Altan, S. Oh and H. Gerel, Metal forming Fundamentals and Applications, American Society of Metals, Metals Park, 1979.

[3] H.A. Attia, K.M. Ewis, I.H.A. Elmaksoud and N. A. Awad-Allah, Hydromagnetic rotating disk flow of a non-Newtonian fluid with heat transfer and ohmic heating, J. Korean Soc. Ind. Appl. Math. 16 (3), 169-180, 2012.

[4] H.A. Attia, Effect of Hall current on the velocity and temperature distributions of Couette flow with variable properties and uniform suction and injection, Comput. Appl. Math. 28 (2), 195-212, 2009.

[5] A.L. Aboul-Hassan and H.A. Attia, The flow due to rotating disk with hall effect, Phys. Lett. A 228, 286-290, 1997.

[6] E.T. Benton, On the flow due do rotating disk, J. Fluid Mech. 24, 781-800, 1966.

[7] R.D. Cess, Unsteady heat transfer from a rotating disk to fluids with low Prandtl numbers, Appl. Sci. Res. 13 (1), 233-240, 1964.

[8] W.G. Cochran, The flow due to rotating disk, Proc. Cambridge Philos. Soc. 30, 365-375, 1934.

[9] K.R. Cramer and S.I. Pai, Magnetofluid Dynamics for Engineers and Applied Physicists, Scripta Publishing Company, 1973.

[10] L.J. Crane, Flow past a stretching plate, Z. Angew. Math. Phys. 21, 645-647, 1970.

[11] A. Das and B. Sahoo, Non-Newtonian stagnation point flow due to a stretchable rotating disk, Fluid Mech. Res. Int. 2 (2), 73-83, 2018.

[12] D.R. Davies, Heat transfer by laminar flow from a rotating disk at large Prandtl numbers, Quart. J. Mech. Appl. Math. 12, 14-21, 1959.

[13] T. Fang, Flow over a stretchable disk, Phys. Fluids 19, 128105, 2007.

[14] T. Fang, C.F.F. Lee and J. Zhang, The boundary layers of an unsteady incompressible stagnation-point flow with mass transfer, Int J Nonlinear Mech, 46 (7), 942-948, 2011.

[15] E.G. Fisher, Extrusion of Plastics, Wiley, New York, 1976.

[16] E.H. Hall, On a New Action of the Magnet on Electric Currents, Amer. J. Math. JSTOR. 2 (3), 287, 1879.

[17] T. Hayatab, S. Qayyuma, M. Imtiaza and A. Alsaedib Flow between two stretchable rotating disks with Cattaneo-Christov heat flux model, Results Phys. 7, 126-133, 2017.

[18] M.A. Hossain, A. Hossain and M. Wilson, Unsteady flow of viscous incompressible fluid with tempreture-dependent viscosity due to a rotating disk in the presence of transverse magnetic field and heat transfer, Int. J. Therm. Sci. 40, 11-20, 2001.

[19] H.A. Jasmine and J.S.B. Gajjar, Convective and absolute instability in the incompressible boundary layer on a rotating disk in the presence of a uniform magnetic field, J. Engrg. Math. 52 (4), 337-353, 2005. 
[20] T.V. Kármán, Uber laminare und turbulente Reibung, ZAMM Z. Angew. Math. Mech. 1, 233-252, 1921.

[21] S.K. Kumar, W.I. Thacker and L.T. Watson, Magnetohydrodynamic flow and heat transfer about a rotating disk with suction and injection at the disk surface, Comput. Fluids, 16 (2), 183-193, 1988.

[22] P.A Laplante, Dictionary of Computer Science, Engineering and Technology CRC Press, 2014

[23] K. Millsaps and K. Pohlhausen, Heat transfer by laminar flow from a rotating plate, J. Aeronaut. Sci. 19, 120-126, 1952.

[24] A. Mushtaq and M. Mustafa, Computations for nanofluid flow near a stretchable rotating disk with axial magnetic field and convective conditions, Results Phys. 7, 3137-3144, 2017.

[25] P. Ram and V. Kumar, FHD flow with heat transfer over a stretchable rotating disk, Multidiscip. Model. Mater. Struct. 9 (4), 524-537, 2013.

[26] N. Riley, The heat transfer from a rotating disk., Quart. J. Mech. Appl. Math. 17, 331-349, 1964.

[27] B. Sahoo, Effects of partial slip, viscous dissipation and Joule heating on Von Kármán flow and heat transfer of an electrically conducting non-Newtonian fluid, Commun. Nonlinear Sci. Numer. Simul. 14, 2982-2998, 2009.

[28] A.M. Siddiqui, M.A. Rana and N. Ahmed, Effects of Hall current and heat transfer on MHD flow of a Burgers' fluid due to a pull of eccentric rotating disks, Commun. Nonlinear Sci. Numer. Simul. 13 (8), 1554-1570, 2008.

[29] C. Soares, Gas Turbines (Second Edition) A Handbook of Air, Land and Sea Applications, 2015.

[30] E.M. Sparrow and R.D. Cess, Magnetohydrodynamic flow and heat transfer about a rotating disk, Trans. ASME Ser. E. J. Appl. Mech. 29, 181-192, 1962.

[31] E.M. Sparrow and J.L. Gregg, Nonsteady surface temperature effects on forced convection heat transfer, J. Aero. Sci. 24, 776-777, 1957.

[32] Z. Tadmor and I. Klein, Engineering principles of plasticating extrusion, Polym. Sci. Eng. Series, New York, Van Norstrand Reinhold, 1970.

[33] M. Turkyilmazoglu, Effects of uniform radial electric field on the MHD heat and fluid flow due to a rotating disk, Internat. J. Engrg. Sci. 51, 233-240, 2012.

[34] M. Turkyilmazoglu, A class of exact solutions for the incompressible viscous magnetohydrodynamic flow over a porous rotating disk, Acta Mech. Sin. 28 (2), 335-347, 2012.

[35] M. Turkyilmazoglu, The MHD boundary layer flow due to a rough rotating disk, ZAMM Z. Angew. Math. Mech. 90 (1), 72-82, 2010.

[36] M. Turkyilmazoglu, Resonance instabilities in the boundary-layer flow over a rotating disk under the influence of a uniform magnetic field, J. Engrg. Math. 59 (3), 337-350, 2007.

[37] M. Turkyilmazoglu, MHD fluid flow and heat transfer due to a stretching rotating disk, Int. J. Therm. Sci. 51, 195-201, 2012.

[38] N. Uygun, Effect of Hall current on the MHD Fluid Flow and Heat Transfer due to a rotating disk with uniform radial electric field, Hacet. J. Math. Stat. 46 (6), 1445-1462, 2015.

[39] N. Uygun, Effects of uniform radial electric field on the MHD and heat transfer due to a shrinking/streching rotating disk, SAUJS, 23 (4), 588-599, 2019.

[40] C.Y. Wang, The three-dimensional flow due to a stretching flat surface, Phys Fluids 27, 1915-1917, 1984.

[41] L.T. Watson and C.Y. Wang, Deceleration of a rotating disk in a viscous fluid, Phys. Fluids, 22 (12), 2267-2269, 1979. 